\title{
Generalization of Schwinger-Zwanziger Dyon to Quaternion
}

\author{
O. P. S. Negi ${ }^{(1,2) *}$, H. Dehnen ${ }^{(1)}$, Gaurav Karnatak ${ }^{(2)}$ and P. S. Bisht ${ }^{(2)}$
}

May 30, 2018

(1) Universität Konstanz

Fachbereich Physik

Postfach-M 677

D-78457 Konstanz, Germany

${ }^{(2)}$ Department of Physics, Kumaun University,

S. S. J. Campus, Almora-263601(Uttarakhand) India

Email-ops_negi@yahoo.com

Heinz.Dehnen@uni-konstanz.de

gauravkarnatak2009@yahoo.in

ps_bisht123@rediffmail.com

\begin{abstract}
Postulating the existence of magnetic monopole in electromagnetism and Heavisidian monopoles in gravitational interactions, a unified theory of gravi-electromagnetism has been developed on generalizing the Schwinger-Zwanziger formulation of dyon to quaternion in simple and consistent manner. Starting with the four Lorentz like forces on different charges, we have generalized the Schwinger-Zwanziger quantization parameters in order to obtain the angular momentum for unified fields of dyons and gravito-dyons (i.e. Gravi-electromagnetism). Taking the unified charge as quaternion, we have reformulated manifestly covariant and consistent theory for the dynamics of four charges namely electric, magnetic, gravitational and Heavisidian associated with gravi electromagnetism.
\end{abstract}

PACS No: $14.80 \mathrm{Hv}$.

${ }^{*}$ Address from November 08- December 22, 2010:- Universität Konstanz, Fachbereich Physik, Postfach-M 677, D-78457 Konstanz, Germany 


\section{Introduction}

The question of existence of monopole [1] and dyons [2] has become a challenging new frontier and the object of more interest in high energy physics. Dirac showed [1] that the quantum mechanics of an electrically charged particle of charge $e$ and a magnetically charged particle of charge $g$ is consistent only if $e g=2 \pi n, n$ being an integer. Schwinger-Zwanziger 2] generalized this condition to allow for the possibility of particles (dyons) that carry both electric and magnetic charge. A quantum mechanical theory can have two particles of electric and magnetic charges $\left(e_{1}, g_{1}\right)$ and $\left(e_{2}, g_{2}\right)$ only if $e_{1} g_{2}-e_{2} g_{1}=2 \pi n$. The angular momentum in the field of the two particle system can be calculated readily with the magnitude $\frac{e_{1} g_{2}-e_{2} g_{1}}{4 \pi c}$. This has an integer or half-integer value, as expected in quantum mechanics, only if $e_{1} g_{2}-$ $e_{2} g_{1}=2 \pi n \hbar c$. The fresh interests in this subject have been enhanced by 't Hooft -Polyakov [3] with the idea that the classical solutions having the properties of magnetic monopoles may be found in Yang - Mills gauge theories. Julia and Zee [4] extended the 't Hooft-Polyakov theory [3] of monopoles and constructed the theory of non Abelian dyons. The quantum mechanical excitation of fundamental monopoles include dyons which are automatically arisen from the semi-classical quantization of global charge rotation degree of freedom of monopoles. In view of the explanation of CP-violation in terms of non-zero vacuum angle of world [5], the monopoles are necessary dyons and Dirac quantization condition permits dyons to have analogous electric charge. Accordingly, a self consistent and manifestly covariant theory has been developed [6] for the generalized electromagnetic fields of dyons.

On the other hand, the analogy between linear gravitational and electromagnetic fields leads to the asymmetry in Einstein's linear equation of gravity and suggests the existence of gravitational analogue of magnetic monopole [7]. Cattani [7] has also derived the covariant field equations for linear gravitation like Maxwell's equations on introducing a new (called the Heavisidian ) field ( like magnetic field ) which depends upon the velocity of gravitational charges (masses). Avoiding the use of arbitrary string variable, the manifestly covariant and consistent theory of gravito - dyons has also been developed [8] in terms of two fourpotentials leading to the structural symmetry between generalized electromagnetic fields of dyons and generalized gravito-Heavisidian fields of gravito-dyons. Extending this recently, a consistent theory for the dynamics of four charges (masses) (namely electric, magnetic, gravitational, Heavisidian) have also been formulated [9] in simple, compact and consistent manner. Considering an invariant Lagrangian density and its quaternionic representation, the consistent field equations for the dynamics of four charges have already been derived [10] and it has been shown that the present reformulation reproduces the dynamics of individual charges (masses) in the absence of other charge (masses) as well as the generalized theory of dyons (gravito - dyons) in the absence gravito - dyons (dyons).

Postulating the existence of magnetic monopole in electromagnetism and Heavisidian monopoles in gravitational interactions, in the present paper, a unified theory of gravielectromagnetism has been developed on generalizing the Schwinger-Zwanziger formulation of dyon to quaternion in simple and consistent manner. Starting with the four Lorentz like forces on different charges, we have generalized the Schwinger-Zwanziger quantization param- 
eters in order to obtain the angular momentum for unified fields of dyons and gravito-dyons (i.e. Gravi-electromagnetism). Taking the unified charge as quaternion, we have reformulated manifestly covariant and consistent theory for the dynamics of four charges namely electric, magnetic, gravitational and Heavisidian associated with gravi electromagnetism. It has been shown that the combined theory describes the interaction of particles in terms of four coupling parameters which we name as the different chirality parameters associated to electric, magnetic, gravitational, and Heavisidian charges. On applying the various quaternion conjugations, it has also been emphasized that the combined theory of gravitation and electromagnetism reproduces the dynamics of generalized electromagnetic (gravito-Heavisidian) fields of dyons (gravito-dyons) in the absence generalized gravito Heavisidian (electromagnetic) fields of gravito-dyons( dyons) or vice versa.

\section{Schwinger-Zwanziger Dyon in Electromagnetic Fields}

Let us consider the case of a Schwinger-Zwanziger dyon which is described as a particle of mass $M$ carrying simultaneously the electric charge $e_{1}$ and magnetic charge. Let this particle moves with velocity $\vec{v}$ so that the force $\overrightarrow{F_{e}}$ experienced by an electric charge is described as,

$$
\overrightarrow{F_{e}}=M \frac{d \vec{v}}{d t}=e_{1}[\vec{E}+\vec{v} \times \vec{H}]
$$

where $\vec{E}$ and $\vec{H}$ are respectively the the electric and magnetic fields and let us take throughout out the notation the system of natural units for which $c=\hbar=1$. Postulation of existence of magnetic monopoles immediately leads to the the following set of symmetric generalized Dirac Maxwell's (GDM) equations[1, 2, 6, 8, 9, 10],

$$
\begin{gathered}
\vec{\nabla} \cdot \vec{E}=\rho_{e} \\
\vec{\nabla} \cdot \vec{H}=\rho_{m} \\
\vec{\nabla} \times \vec{E}=-\frac{\partial \vec{H}}{\partial t}-\overrightarrow{j_{m}} \\
\vec{\nabla} \times \vec{H}=\overrightarrow{j_{e}}+\frac{\partial \vec{E}}{\partial t}
\end{gathered}
$$

where $\rho_{e}$ and $\rho_{m}$ are respectively the electric and magnetic charge densities while $\overrightarrow{j_{e}}$ and $\overrightarrow{j_{m}}$ are respectively the electric and magnetic current densities. These GDM equations are invariant not only under Lorentz and conformal transformations but also invariant under the following duality transformations between electric $\mathcal{E}$ and magnetic $\mathcal{H}$ quantities i.e.

$$
\begin{aligned}
\mathcal{E} & \Longrightarrow \mathcal{E} \cos \vartheta+\mathcal{H} \sin \vartheta \\
\mathcal{H} & \Longrightarrow \mathcal{H} \cos \vartheta-\mathcal{E} \sin \vartheta
\end{aligned}
$$


where $\mathcal{E}=\left(e_{1}, \vec{E}, \rho_{e}, \overrightarrow{j_{e}}\right)$ and $\mathcal{H}=\left(g_{1}, \vec{H}, \rho_{m}, \overrightarrow{j_{m}}\right)$. For a particular value of $\vartheta=\frac{\pi}{2}$, equations (3) reduces to

$$
\mathcal{E} \longmapsto \mathcal{H} \quad \mathcal{H} \longmapsto-\mathcal{E}
$$

Accordingly the force experienced by a magnetic monopole can be written by applying the duality transformations $\vec{E} \longrightarrow \vec{H}, \vec{H} \longrightarrow-\vec{E}$ and $e_{1} \longmapsto g_{1}$ as,

$$
\overrightarrow{F_{g}}=g_{1}[\vec{H}-\vec{v} \times \vec{E}]
$$

Schwinger-Zwangiger[2] generalized equations (11) and (5) as the equation of motion for the force experienced by a particle (dyon) carrying simultaneous existence of electric and magnetic charges as

$$
\vec{F}=M \frac{d \vec{v}}{d t}=\vec{F}_{e}+\overrightarrow{F_{g}}=e_{1}[\vec{E}+\vec{v} \times \vec{H}]+g_{1}[\vec{H}-\vec{v} \times \vec{E}]
$$

where we may write the following forms of the electric and magnetic field strengths at the point with $\vec{r}$ of magnitude $r$,

$$
\vec{E}=e_{2} \frac{\vec{r}}{r^{3}}, \text { and } \vec{H}=g_{2} \frac{\vec{r}}{r^{3}}
$$

in which the electric $e_{2}$ and magnetic $g_{2}$ charges for a stationary body are located at origin. Substituting $\vec{E}$ and $\vec{H}$ from equation (7) into the equation (6), we get the following expression for equation of motion for Schwinger-Zwangiger [2] dyon as

$$
\vec{F}=M \frac{d \vec{v}}{d t}=\alpha_{12} \frac{\vec{r}}{r^{3}}+\beta_{12} \frac{\vec{r} \times(\vec{v} \times \vec{r})}{r^{3}}
$$

where

$$
\alpha_{12}=\left(e_{1} e_{2}+g_{1} g_{2}\right) \quad \text { and } \quad \beta_{12}=\left(e_{1} g_{2}-g_{1} e_{2}\right)
$$

are respectively known as electric and magnetic coupling parameters of dyons. Taking the vector product of equation (8) with $\vec{r}$, we get

$$
\vec{r} \times M \frac{d \vec{v}}{d t}=\alpha_{12} \frac{\overrightarrow{(r} \times \vec{r})}{r^{3}}+\beta_{12} \frac{\vec{r} \times(\vec{v} \times \vec{r})}{r^{3}}
$$


and using vector multiplication rule $\vec{r} \times \vec{r})=0$ and $\frac{d}{d t}(\vec{r} \times M \vec{v})=\vec{r} \times M \frac{d \vec{v}}{d t}$, the equation (10) reduces to,

$$
\frac{d}{d t}(\vec{r} \times M \vec{v})=\beta_{12} \frac{\vec{r} \times(\vec{v} \times \vec{r})}{r^{3}}=\beta_{12} \frac{d}{d t}\left(\frac{\vec{r}}{r}\right)
$$

where we have used the identity $\frac{\vec{r} \times(\vec{v} \times \vec{r})}{r^{3}}=\frac{d}{d t}\left(\frac{\vec{r}}{r}\right)$. So that we may define following expression for conserved angular momentum for dyon as

$$
\vec{J}=\vec{r} \times M \vec{v}-\beta_{12} \frac{\vec{r}}{r}
$$

which gives rise the component of the angular momentum $\vec{J}$ along the direction of $\vec{r}$ as

$$
J_{r}=\hat{r} \cdot \vec{J}=\beta_{12} \hat{r} \cdot \frac{\vec{r}}{r}=\beta_{12}
$$

where $\hat{r}$ is the unit vector along the vector $\vec{r}$ and we have used $\overrightarrow{(r} \times \vec{r})=0$. Thus the quantization of the component of the angular momentum $J_{r}$ along the line of particle leads to Schwinger-Zwangiger 2] charge quantization ( or chirality quantization) in the units of Plank's constant

$$
\beta_{12}=\left(e_{1} g_{2}-g_{1} e_{2}\right)=\nu
$$

where $\nu$ may be an integer or half integer. But in most cases, it has been taken as integer as its half integral values were already excluded by Dirac in his seminal paper[1]. On substituting $e_{1}=e ; g_{1}=0 ; e_{2}=0$ and $g_{2}=g$ in equation (14) for interaction of two dyons with charges $(e, 0)$ and $(0, g)$, we get the Dirac quantization condition[1]

$$
e g=\nu
$$

in the units of Plank constant. It is clear that if we do not consider dyon Dirac quantization condition is not dual invariant.

\section{Schwinger-Zwanziger Dyon in Gravito-Heavisidian Fields}

Analogy between electromagnetic and linear gravitational field equations suggests [7] the structural symmetry between these two forces of nature. Accordingly, on postulating the existence of Heavisidian monopole [11] leads the symmetry between the linear equations of gravito-Heavisidian fields and the generalized Maxwell's Dirac equations in electromagnetic 
Fields. Likewise, generalized Maxwell's Dirac equations (2), we may write the the following form [8, 11] of linear equations for generalized gravito- Heavisidian fields in presence of Heavisidian monopole as

$$
\begin{gathered}
\vec{\nabla} \cdot \overrightarrow{\mathrm{G}}=-\rho_{g} \\
\vec{\nabla} \cdot \overrightarrow{\mathrm{H}}=-\rho_{h} \\
\vec{\nabla} \times \overrightarrow{\mathrm{G}}=-\frac{\partial \overrightarrow{\mathrm{H}}}{\partial t}+\overrightarrow{j_{h}} \\
\vec{\nabla} \times \overrightarrow{\mathrm{H}}=\overrightarrow{j_{g}}+\frac{\partial \overrightarrow{\mathrm{G}}}{\partial t}
\end{gathered}
$$

where $\vec{G}$ is the gravitational (gravi-electric) field, $\overrightarrow{\mathrm{H}}$ is the Heavisidian (gravi-magnetic) field, $\rho_{g}$ is the gravitational charge (mass) density, $\rho_{h}$ is Heavisidian charge (mass) density, $\overrightarrow{j_{g}}$ is gravitational current density and $\overrightarrow{j_{h}}$ denotes the Heavisidian current density. Similarly, the force $\overrightarrow{F_{m}}$ the force experienced by a gravitational (gravi-electric) charge (mass) $m_{1}$ may then be expressed as ,

$$
\overrightarrow{F_{m}}=m_{1} \frac{d \vec{v}}{d t}=m_{1}[\overrightarrow{\mathrm{G}}-\vec{v} \times \overrightarrow{\mathrm{H}}]
$$

Accordingly GDM type equations of gravito-Heavisidian fields are invariant not only under Lorentz and conformal transformations but also invariant under the following duality transformations between gravitational (gravi-electric) $\mathcal{G}$ and Heavisidian (gravi-magnetic) $\mathcal{M}$ quantities i.e

$$
\begin{aligned}
\mathcal{G} & \Longrightarrow \mathcal{G} \cos \vartheta+\mathcal{M} \sin \vartheta \\
\mathcal{M} & \Longrightarrow \mathcal{M} \cos \vartheta-\mathcal{G} \sin \vartheta
\end{aligned}
$$

where $\mathcal{G}=\left(m_{1}, \overrightarrow{\mathrm{G}}, \rho_{g}, \overrightarrow{j_{g}}\right)$ and $\mathcal{M}=\left(h_{1}, \overrightarrow{\mathrm{H}}, \rho_{h}, \overrightarrow{j_{h}}\right)$ with $h_{1}$ denotes the Heavisidian (gravimagnetic) charge (mass). For a particular value of $\vartheta=\frac{\pi}{2}$, equations (18) reduces to

$$
\mathcal{G} \longmapsto \mathcal{M} \mathcal{M} \longmapsto-\mathcal{G}
$$

So, on applying the duality symmetry on gravitational and Heavisidian fields and charges (masses) like the electromagnetism, we may write the net force experienced by Heavisidian (gravi-magnetic) charge (mass) $h_{1}$ as ,

$$
\overrightarrow{F_{h}}=h_{1} \frac{d \vec{v}}{d t}=h_{1}[\overrightarrow{\mathrm{H}}+\vec{v} \times \vec{G}] .
$$


Following Schwinger-Zwangiger[2], here also we may adopt the same process for gravito-dyons. Hence the gravito dyons are considered as the particles carrying simultaneously the existence of gravitational (gravi-electric) and Heavisidian (gravi-magnetic) charges (masses). Thus, we may write the net force $\vec{F}$ acting on gravito-dyon [11] as

$$
\overrightarrow{\mathrm{F}}=M \frac{d \vec{v}}{d t}=\left(m_{1}+h_{1}\right) \frac{d \vec{v}}{d t}=m_{1}[\overrightarrow{\mathrm{G}}-\vec{v} \times \overrightarrow{\mathrm{H}}]+h_{1}[\overrightarrow{\mathrm{H}}+\vec{v} \times \vec{G}]
$$

where following expressions for stationary gravitational (gravi-electric) and Heavisidian (gravimagnetic) field strengths at the point with $\vec{r}$ of magnitude $r$ may be used with gravitational charge(mass) $m_{2}$ and Heavisidian charge(mass) $h_{2}$ as

$$
\overrightarrow{\mathrm{G}}=m_{2} \frac{\vec{r}}{r^{3}} \text { and } \overrightarrow{\mathrm{H}}=h_{2} \frac{\vec{r}}{r^{3}}
$$

Substituting $\vec{G}$ and $\vec{H}$ from equation (22) into the equation (21), we get the following expression for equation of motion for Schwinger-Zwangiger [2] gravito-dyon as

$$
\overrightarrow{\mathrm{F}}=M \frac{d \vec{v}}{d t}=\left(m_{1}+h_{1}\right) \frac{d \vec{v}}{d t}=\gamma_{12} \frac{\vec{r}}{r^{3}}+\delta_{12} \frac{\vec{r} \times(\vec{v} \times \vec{r})}{r^{3}}
$$

where

$$
\gamma_{12}=\left(m_{1} m_{2}+h_{1} h_{2}\right) \quad \text { and } \quad \delta_{12}=\left(m_{1} h_{2}-h_{1} m_{2}\right)
$$

are respectively known as and gravitational (gravi-electric) and Heavisidian (gravi-magnetic) coupling parameters of gravito-dyons. Taking the vector product of equation (23) with $\vec{r}$, we get

$$
\vec{r} \times\left(m_{1}+h_{1}\right) \frac{d \vec{v}}{d t}=\gamma_{12} \frac{\overrightarrow{(r} \times \overrightarrow{r)}}{r^{3}}+\delta_{12} \frac{\vec{r} \times(\vec{v} \times \vec{r})}{r^{3}}
$$

Using vector multiplication rule $\overrightarrow{(r} \times \vec{r})=0$ and $\frac{d}{d t}\left[\vec{r} \times\left(m_{1}+h_{1}\right) \vec{v}\right]=\vec{r} \times\left(m_{1}+h_{1}\right) \frac{d \vec{v}}{d t}$, the equation (25) reduces to,

$$
\frac{d}{d t}\left[\vec{r} \times\left(m_{1}+h_{1}\right) \vec{v}\right]=\delta_{12} \frac{\vec{r} \times(\vec{v} \times \vec{r})}{r^{3}}=\delta_{12} \frac{d}{d t}\left(\frac{\vec{r}}{r}\right)
$$

where $\frac{\vec{r} \times(\vec{v} \times \vec{r})}{r^{3}}=\frac{d}{d t}\left(\frac{\vec{r}}{r}\right)$. As such, we may write the following expression for conserved angular momentum for gravito-dyon as

$$
\overrightarrow{\mathcal{J}}=\vec{r} \times\left(m_{1}+h_{1}\right) \vec{v}-\delta_{12} \frac{\vec{r}}{r}
$$


which gives rise the component of the angular momentum $\vec{J}$ along the direction of $\vec{r}$ as

$$
\mathcal{J}_{r}=\hat{r} \cdot \vec{J}=\delta_{12} \hat{r} \cdot \frac{\vec{r}}{r}=\delta_{12}
$$

where $\hat{r}$ is the unit vector along the vector $\vec{r}$ and $\overrightarrow{(r} \times \vec{r})=0$. Thus the quantization of the component of the angular momentum $J_{r}$ along the line of particle leads to SchwingerZwangiger [2] charge (mass) quantization ( or chirality quantization) condition for gravitodyons in the units of Plank's constant

$$
\beta_{12}=\left(e_{1} g_{2}-g_{1} e_{2}\right)=\mathrm{n}
$$

where $\mathrm{n}$ may be an integer or half integer as may be the case of dyons in electromagnetic fields.. On substituting $m_{1}=m ; h_{1}=0 ; m_{2}=0$ and $h_{2}=h$ in equation (29) for interaction of two gravito-dyons with charges (masses) $(m, 0)$ and $(0, h)$, we get the Dirac quantization condition[1] for gravito-dyons as

$$
m h=\mathrm{n}
$$

which can be dual invariant only if we consider the case of gravito-dyons in linear gravitational fields.

\section{Generalization Schwinger-Zwanziger Dyon to Quaternion}

Let us assume that a particle of mass $M$ carries simultaneous existence of four charges namely electric $\left(e_{1}\right)$, magnetic $\left(g_{1}\right)$, gravitational (gravi-electric) $\left(m_{1}\right)$ and Heavisidian (gravimagnetic) $\left(h_{1}\right)$. Let this particle moves with velocity $\vec{v}$ so that it experiences a combined force which is the sum of the forces exerted independently due to individual charges i.e.

$$
\overrightarrow{\boldsymbol{F}}=\overrightarrow{F_{e}}+\overrightarrow{F_{g}}+\overrightarrow{F_{m}}+\overrightarrow{F_{h}}
$$

where $\overrightarrow{F_{e}}, \overrightarrow{F_{g}}, \vec{F}_{g}$ and $\vec{F}_{h}$ are respectively given by equations (11), (5), (17) and (20). Now substituting the values of electric $(\vec{E})$, magnetic $(\vec{H})$, gravitational (gravi-electric) $(\vec{G})$ and Heavisidian (gravi-magnetic) $(\vec{H})$ field strengths given by equations (7), and (8) in to equation (31), we get

$$
\overrightarrow{\boldsymbol{F}}=W_{12} \frac{\vec{r}}{r^{3}}+\left(X_{12}+Y_{12}+Z_{12}\right) \vec{v} \times \frac{\vec{r}}{r^{3}}
$$

where 


$$
\begin{aligned}
W_{12} & =\left(e_{1} e_{2}+g_{1} g_{2}+m_{1} m_{2}+h_{1} h_{2}\right) ; \\
X_{12} & =\left(e_{1} g_{2}-e_{2} g_{1}+m_{1} h_{2}-m_{2} h_{1}\right) ; \\
Y_{12} & =\left(e_{1} m_{2}-m_{1} e_{2}-h_{2} g_{1}+h_{1} g_{2}\right) ; \\
Z_{12} & =\left(e_{1} h_{2}-h_{1} e_{2}+g_{1} m_{2}-m_{1} g_{2}\right) .
\end{aligned}
$$

are different four coupling parameters. $W_{12}, X_{12}, Y_{12}, Z_{12}$ may also be identified as electric, magnetic, gravitational and Heavisidian parameters [9]. According to the Newton's second law $\vec{F}=M \frac{d \vec{v}}{d t}$, equation (32) is written as

$$
M \frac{d \vec{v}}{d t}=W_{12} \frac{\vec{r}}{r^{3}}+\left(X_{12}+Y_{12}+Z_{12}\right) \vec{v} \times \frac{\vec{r}}{r^{3}}
$$

Taking the vector product of equation (37) with $\vec{r}$, we get

$$
\vec{r} \times M \frac{d \vec{v}}{d t}=W_{12} \frac{\overrightarrow{(r} \times \vec{r})}{r^{3}}+\left(X_{12}+Y_{12}+Z_{12}\right) \frac{\vec{r} \times(\vec{v} \times \vec{r})}{r^{3}}
$$

Using vector multiplication rule $\overrightarrow{(r} \times \vec{r})=0, \frac{d(\vec{r} \times M \vec{v})}{d t}=\vec{r} \times m \frac{d \vec{v}}{d t}$, and the identity $\frac{\vec{r} \times(\vec{v} \times \vec{r})}{r^{3}}=\frac{d}{d t}\left(\frac{\vec{r}}{r}\right)$, equation (38) reduces to ,

$$
\frac{d}{d t}(\vec{r} \times M \vec{v})=\left(X_{12}+Y_{12}+Z_{12}\right) \frac{d}{d t}\left(\frac{\vec{r}}{r}\right) .
$$

Thus by adopting the above procedure, we get the following expression for angular momentum $\vec{J}$ as

$$
\vec{J}=\vec{r} \times M \vec{v}-\left(X_{12}+Y_{12}+Z_{12}\right)\left(\frac{\vec{r}}{r}\right)
$$

which gives rise the component of the angular momentum $\vec{J}$ along the direction of $\vec{r}$ as,

$$
\mathrm{J}_{r}=\left(X_{12}+Y_{12}+Z_{12}\right)
$$

This is called the residual component of unified angular momentum and leads the following form of generalized Schwinger-Zwangiger [2] quantization condition i.e

$$
\mathrm{J}_{r}=\left(X_{12}+Y_{12}+Z_{12}\right)=n
$$

where $n$ is an integer and $h$ is Planck's constant. 


\section{Quaternion Formulation for Gravi-Electromagnetism}

A unified theory of generalized electromagnetic and Heavisidian fields may then be developed consistently by generalizing Schwinger-Zwangiger [2] dyon to a quaternion possessing a quartet $(e, g, m, h)$ of four charges. Quaternion $(e, g, m, h)$ charge thus represents the theory of gravi-electromagnetism for the particles carrying simultaneously electric, magnetic, gravitational and Heavisidian charges. So, let us generalize two types of Schwinger-Zwangiger [2] dyonic charges $(e, g)$ and $(m, h)$ to a quaternion charge of gravi- electromagnetic fields as [8] as,

$$
Q=(e-i g)-j(m-i h)=e-i g-j m-k h
$$

where $i, j, k$ are three quaternion basis elements satisfying the quaternion multiplication rules 8

$$
\begin{gathered}
i \cdot i=j \cdot j=k \cdot k=-1 \\
i \cdot j=-j \cdot i=k \\
j \cdot k=-k \cdot j=i \\
k \cdot i=-i \cdot k=j .
\end{gathered}
$$

The quaternion conjugation of equation (43) is defined as

$$
\bar{Q}=(e+i g)+(m+i h) j=e+i g+j m+k h .
$$

Thus, on using the quaternion multiplication rule (44), the interaction of between two quaternions $(a$ and $b)$ with charges $Q_{a}=\left(e_{a}, g_{a}, m_{a}, h_{a}\right)=e_{a}-i g_{a}-j m_{a}-k h_{a}$ and $Q_{b}=$ $\left(e_{b}, g_{b}, m_{b}, h_{b}\right)=e_{b}-i g_{b}-j m_{b}-k h_{b}$ leads [9] to

$$
\begin{aligned}
& \overline{Q_{a}} Q_{b}=\left(e_{a}+i g_{a}+j m_{a}+k h_{a}\right)\left(e_{b}-i g_{b}-j m_{b}-k h_{b}\right) \\
& \quad=\alpha_{a b}+\beta_{a b}+\gamma_{a b}+\delta_{a b}
\end{aligned}
$$

where

$$
\begin{aligned}
\alpha_{a b} & =\left(e_{a} e_{b}+g_{a} g_{b}+m_{a} m_{b}+h_{a} h_{b}\right) ; \\
\beta_{a b} & =\left(e_{a} g_{b}-e_{b} g_{a}+m_{a} h_{b}-m_{b} h_{a}\right) ; \\
\gamma_{a b} & =\left(e_{a} m_{b}-m_{a} e_{b}-h_{b} g_{a}+h_{a} g_{b}\right) ; \\
\delta_{a b} & =\left(e_{a} h_{b}-h_{a} e_{b}+g_{a} m_{b}-m_{a} g_{b}\right) .
\end{aligned}
$$


Hence,for $a=1$ and $b=2$, equations (47- 50) are same as equations (33- 36) for four different chirality parameters i.e $\alpha_{12}=W_{12}, \beta_{12}=X_{12}, \gamma_{12}=Y_{12}$, and $\delta_{12}=Z_{12}$. So, the equations (33) and (34) immediately reduces to $W_{12}=e_{1} e_{2}+g_{1} g_{2}$ and $X_{12}=\left(e_{1} g_{2}-e_{2} g_{1}\right)$ for the interaction of two Schwinger-Zwangiger [2] dyons from two quaternion charges $\left(e_{1}, g_{1}, 0,0\right)$ and $\left(e_{2}, g_{2}, 0,0\right)$ with the vanishing of other parameters. Similarly, for the Schwinger-Zwangiger case for interaction of gravito-dyons i.e. gravitational charge and Heavisidian monopole , we get $W_{12}=m_{1} m_{2}+h_{1} h_{2}$ and $X_{12}=m_{1} h_{2}-h_{1} m_{2}$ from quaternions $\left(0,0, m_{1}, h_{1}\right)$ and $\left(0,0, m_{2}, h_{2}\right)$. Similarly, we may be speculate a new kind of dyon i.e. electric charge and Heavisidian monopole obtained from a quaternion like $(e, 0,0, h)$ where we may get $W_{12}=$ $e_{1} e_{2}+h_{1} h_{2}$ and $Z_{12}=e_{1} h_{2}-h_{1} e_{2}$. Similarly a new kind of dyon i.e., electric charge and gravitational charge may also be speculated from the quaternion like $(e, 0, m, 0)$ for which we get $W_{12}=e_{1} e_{2}+m_{1} m_{2}$ and $Y_{12}=e_{1} m_{2}-m_{1} e_{2}$. Also there are the possibilities of other dyons like magnetic charge and gravitational charge from a quaternion $(0, g, m, 0)$ for which $W_{12}=m_{1} m_{2}+g_{1} g_{2}$ and $Z_{12}=g_{1} m_{2}-m_{1} g_{2}$ as well as for the purely hypothetical dyons like magnetic and Heavisidian monopoles we get $W_{12}=h_{1} h_{2}+g_{1} g_{2}$ and $Y_{12}=h_{1} g_{2}-g_{1} h_{2}$. As such, the quaternion generalization of Schwinger-Zwangiger [2] quantization condition of dyons extends the possibilities of six types of dyons like $(e, g),(m, h),(e, h),(e, h),(g, m)$ and $(g, h)$. Let us try to obtain the six kinds of Schwinger-Zwangiger dyons from a quaternion (43).

- Applying the $j$-conjugation i.e. $i \rightarrow i, j \rightarrow-j, k \rightarrow-k$ as $k=i j$ on quaternion (43), we get,

$$
q^{j}=e-i g+j m+k h
$$

and adding this equations (51) to quaternion (43), we get

$$
e-i g=\frac{1}{2}\left(q+q^{j}\right)
$$

which refers to generalized charge (as complex quantity) as the order pair of $(e, g)$ for Schwinger-Zwangiger dyon moving in electromagnetic fields.

- Similarly, on subtracting equation (51) from equation (43), we get

$$
m-i h=\frac{1}{2 j}\left(q-q^{j}\right)
$$

which refers to generalized charge (as complex quantity) as the order pair of $(m, h)$ for Schwinger-Zwangiger dyon moving in gravito-Heavisidian fields.

- Now, applying $i$-conjugation i.e $i \rightarrow-i, j \rightarrow j, k \rightarrow-k$ as $k=i j$ on quaternion (43), we get 


$$
q^{i}=e+i g-j m+k h
$$

and adding the this equations (54) to (43) we find

$$
e-j m=\frac{1}{2}\left(q+q^{j}\right)
$$

which defines the generalized charge (as complex quantity) as the order pair of $(e, m)$ for of a Schwinger-Zwangiger dyon obtained from electric and gravitational charges.

- Furthermore, on subtracting equation (25) from equation (21), we get

$$
(g-j h)=\frac{1}{2 i}\left(q-q^{i}\right)
$$

which describes the generalized charge (as complex quantity) as the order pair of $(g, h)$ for of a Schwinger-Zwangiger dyon obtained from magnetic and Heavisidian charges.

- Applying the transformation for $i \rightarrow-i, j \rightarrow j, k \rightarrow-k$ in equation (43), we get,

$$
q^{k}=e+i g+j m-k h
$$

and adding equations (43) and (57), we get

$$
e-k h=\frac{1}{2}\left(q+q^{k}\right)
$$

which gives the generalized charge (as complex quantity) as the order pair of $(e, h)$ for of a Schwinger-Zwangiger dyon obtained from from electric and Heavisidian charges.

- Similarly on subtracting the equation (57) from equation (43), we find,

$$
q-q^{k}=-2(i g+j m)
$$

and applying the quaternion property $j=-i k$, the equation (59) reduces to ,

$$
(g-k m)=\frac{i\left(q-q^{k}\right)}{2}
$$


which describes the generalized charge (as complex quantity) as the order pair of $(g, m)$ for of a Schwinger-Zwangiger dyon obtained from magnetic and gravitational charges.

Acknowledgment: One of us OPSN expresses his thanks of gratitude to German Academic Exchange Service (Deutscher Akademischer Austausch Dienst), Bonn for their financial support under DAAD re-invitation programme at Universität Konstanz.

\section{References}

[1] P. A. M. Dirac, Proc. Roy. Soc. London, $\underline{\mathbf{A 1 3 3}}$, (1931), 60 ; Phys. Rev., $\underline{\mathbf{7 4}}$, (1948), 817.

[2] J. Schwinger , Phys. Rev., 144 (1966) 1087; 151 (1966) 1055, 1048; Science, 165 (1969) 757; D. Zwanziger, Phys. Rev., B137 (1965) 647; Phys. Rev.,176 (1968) 1489.

[3] G.' t Hooft, Nucl.Phys., B79 (1974), 276 ; A. M. Polyakov, JETP Lett., 20 , (1974), 194.

[4] B. Julia and A. Zee, Phys Rev, D21 (1980) 2940; M. Prasad and C. Sommerfield, Phys Rev Lett. , $3 \underline{5}$ (1975) 760

[5] E. Witten, Phys. Lett., B86, 283 (1979); J. Preskill, Ann. Rev. Nucl. Sci., 34, 461 (1984).

[6] Jivan Singh, P. S. Bisht and O. P. S. Negi, J. Phys. A: Math. and Theoretical, $\underline{40}$ (2007), 11395; J. Phys. A: Math. and Theoretical, 40 (2007), 9137.

[7] D. D. Cattani, Nuovo Cimento, B60 (1980), 67. L. Motz, Nuovo Cimento, B12 ,239 (1972); L. Motz, Nuovo Cimento, $\underline{\mathbf{A 3 7}}, 13$ (1977); J. S. Dowker and R. A. Roche, Proc. of Phys. Soc., $\underline{92}$ (1967), 1.

[8] P. S. Bisht, O. P. S. Negi and B. S. Rajput, Inter. J. of Theor. Phys., 2, 2099 (1993); Prog. Theor. Phys., $\underline{\mathbf{8 5}}, 157$ (1991).

[9] Shalini Dangwal, P. S. Bisht and O. P. S. Negi, Russian Physics Journal, $\underline{49}$ (2006), 1274; P. S. Bisht, O. P. S. Negi and B. S. Rajput, Indian J. Pure \& Appl. Math., $\underline{\mathbf{3 1}}, 543$ (1993).

[10] Gaurav Karnatak, P. S. Bisht and O. P. S. Negi, Inter. J. of Theor. Phys., $\underline{49}$ (2010), 1344.

[11] A. Singh, Letter Nuovo Cimento, $\underline{\mathbf{3 2}}, 1231$ (1981); $\underline{\mathbf{3 2}}, 5$ (1981); $\underline{\mathbf{3 2}}, 231$ (1981); B.S. Rajput, Letter Nuovo Cimento, $\underline{\mathbf{3 5}}, 205$ (1982). 